\title{
Treatment and outcome of metastatic paraganglioma of the spine
}

\author{
Qi Jia ${ }^{1} \cdot$ Huabin $\mathrm{Yin}^{2} \cdot$ Jian Yang ${ }^{1} \cdot$ Zhipeng $\mathrm{Wu}^{1} \cdot$ Wangjun $\mathrm{Yan}^{1} \cdot$ \\ Wang Zhou ${ }^{1} \cdot$ Xinghai Yang ${ }^{1} \cdot$ Jianru Xiao $^{1}$
}

Received: 2 January 2017/Revised: 11 April 2017/Accepted: 15 May 2017/Published online: 26 June 2017

(C) The Author(s) 2017. This article is an open access publication

\begin{abstract}
Background Spinal metastatic paraganglioma (MPG) is rare and only reported in individual case reports. The low incidence makes it difficult to define appropriate therapy and prognosis. Our study illustrated the largest series to discuss the possible treatment and outcomes of patients with spinal MPG.

Methods A retrospective study of 15 patients with spinal MPG who were surgically treated between 2005 and 2014 was performed. Three surgical modalities were applied, and radiotherapy and chemotherapy were utilized as adjuvant therapy.

Results The mean patients age was 40.9 (range 23-58) years. The period between primary surgery and spinal metastasis averaged $8.2(0.5-15)$ years. Lesions were mainly located in cervical spine (2), thoracic spine (8), lumbar spine (3), and sacrum (2). The mean follow-up period was 35.0 months. Lesion progression was detected in nine patients, whereas five patients (33.3\%) passed away. For solitary spine, multiple bone and both bone and nonosseous metastasis cases, the mean progression-free
\end{abstract}

Qi Jia, Huabin Yin and Jian Yang contributed equally to this work, and all should be considered first author.

Xinghai Yang

cnspineyang@163.com

$\triangle$ Jianru Xiao

jianruxiao83@163.com

1 Department of Orthopedic Oncology, Shanghai Changzheng Hospital, Second Military Medical University, 415 Fengyang Road, Huangpu District, Shanghai, China

2 Department of Orthopedics, Shanghai General Hospital, School of Medicine, Shanghai Jiaotong University, 100 Haining Road, Shanghai, China survival was 41 (range 9-56), 22.5 (range 12-38) and 8.3 (range 3-18) months, respectively.

Conclusions The cases presented in the current study highlight the crucial role of surgery. Total en bloc for solitary spinal MPG could result in a satisfying prognosis and piecemeal total resection with postoperative radiotherapy could be an alternative therapy. Radiotherapy and chemotherapy were advocated, especially for the multiple metastasis.

Keywords Metastatic paraganglioma - Spine . Retrospective study · Total en bloc spondylectomy . Adjuvant therapy

\section{Introduction}

According to the World Health Organization, paraganglioma (PG) is defined as a tumor arising from the paraganglia outside the adrenal medulla [1]. PG, a relatively rare tumor and mainly occurring in the head, neck, thorax, abdomen, and pelvis, accounts for about $0.3 \%$ of all neoplasms with an annual incidence of $1 / 100,000[2,3]$. It is a slow-growing tumor that often presents as a painless mass in the fifth or sixth decade of life [4], and often recurs after incomplete surgical resection. Although regarded as benign in nature, PG possesses metastatic possibility which is a marker of malignant transformation $[5,6]$ as there are no molecular or cellular markers existing for determining whether PG is malignant [7-9]. Metastatic PGs (MPG) are among the solid tumors that most frequently spread to the skeleton, with spine being the most frequently affected site [10].

Spinal MPG was uncommon and sporadically reported in the English literature [2-6, 11-17] with no case series 
study. Spinal lesions could cause bone destruction or compression of the spinal cord, clinically manifesting as pain or neurological deficits. Surgical treatment including subtotal (curettage) or total resection with/without adjuvant radiation was recommended by some authors $[1,2,15]$ which could provide limited information to the treatment of spinal cases. The probably epidemiological and treatment features of spinal MPG are not clear as well. Here, we present our experience of 15 consecutive spinal MPG patients with middle to long-term follow-up, trying to illustrate the clinical features and different treatments and outcomes of spinal MPG.

\section{Materials and methods}

A retrospective review of 15 patients with spinal MPG who were surgically treated in our institution between 2005 and 2014 was performed. Surgery was performed in all patients using different surgical modalities according to the Tokuhashi [17] and Tomita [18] scoring system. We focused on the tumor progression status and overall survival (OS) after the initial surgery in our center, and progression-free survival (PFS) was defined as the interval between the date of surgery and the date of tumor progression or other new metastasis. This study was approved by the Ethics Committee of the hospital, and informed consent was obtained from the surviving patients or family members of those who had passed away.

The clinical information and surgical data of 15 patients are shown in Table 1. All patients had undergone primary surgery with the pathological diagnosis of extra-adrenal PG before admission into our center before. The diagnosis of spinal MPG was confirmed by three criteria: (1) identified on a plain standard X-ray, computed tomography (CT), magnetic resonance imaging (MRI), bone scan, or fluorodeoxyglucose (FDG) positron emission tomography (PET)/CT scan; (2) had the primary PG treatment history; (3) identified in a pathological specimen from a biopsy or open surgery. The preoperative neurologic status was classified according to the Frankel score [20]. The life quality of all patients was assessed with the Karnofsky score system [21]. Surgery through individualized approaches was conducted by the surgery team leaded by professor Xiao. The screw-rod system was used to reconstruct stability of the spine [22], and an anterior titanium mesh and plate was applied to patients with cervical lesions.

Regular radiographic assessment (X-ray, CT or MRI) was performed at 3,6 , and 12 months after surgery, every 6 months for the next 2 years, and then annually for life. For patients showing tumors progression, PET-CT was recommended. Follow-up data were obtained from office visits and telephone interviews. In the follow-up visits at 3 months after surgery, neural function and lift quality were re-evaluated based on the Frankel score system and Karnofsky score system. The follow-up period was defined as the interval from the date of surgery to death, or until September 2016 for patients alive.

\section{Results}

\section{Patient features}

Our series comprised 13 men and 2 women, with an average age of 40.9 (ranging 23-58) years. Lesions were mainly located in cervical spine in two cases, thoracic spine in eight cases, lumbar spine in three cases, and sacrum in two cases. In addition, solitary spine, multiple bone and both bone and nonosseous metastasis occurred in 7, 4 and 4 patients, respectively. Localized pain was the most consistent complaint, with a mean duration of 8.8 months as the preoperative symptom. Additional symptoms included catecholamine-related symptoms with remarkable hypertension $(180 / 110 \mathrm{~mm} \mathrm{Hg})$ in one patient (Case 5), and varying degrees of cord compression at diagnosis in 10 patients.

The mean follow-up period was 35.0 (range 6-118) months. Lesion progression was detected in 9 patients (60\%) with a mean PFS of 19.6 (range 3-56) months after the initial surgery in our center, whereas 5 patients $(33.3 \%)$ passed away with the mean period of 38.2 (range 6-118) months. For solitary spine, multiple bone and both bone and nonosseous metastatic cases, the mean PFS time was 41 (range 9-56), 22.5 (range 12-38) and 8.3 (range 3-18) months, respectively (Fig. 1a).

\section{Treatment history}

Before admission into our center, all patients had undergone total resection of primary tumors locating at the retroperitoneum $(n=10,66.7 \%)$, para-aortic region $(n=2,13.3 \%)$, and upper mediastinum $(n=3,20.0 \%)$. The mean maximum diameter of the lesions in CT images was 6.3 (range 3.5-8.5) $\mathrm{cm}$ with 2 lesions less than $5 \mathrm{~cm}$. The period between previous surgery and spinal metastasis averaged $8.2(0.5-15)$ years (Table 1$)$.

\section{Radiologic study}

Osteolytic change of spinal lesion was common in X-ray radiography and CT scans. MRI is considered to be the superior imaging modality for extra-adrenal PG [16], but it cannot specifically distinguish spinal MPG from other spinal malignant tumors. In our series, giant cell tumors 


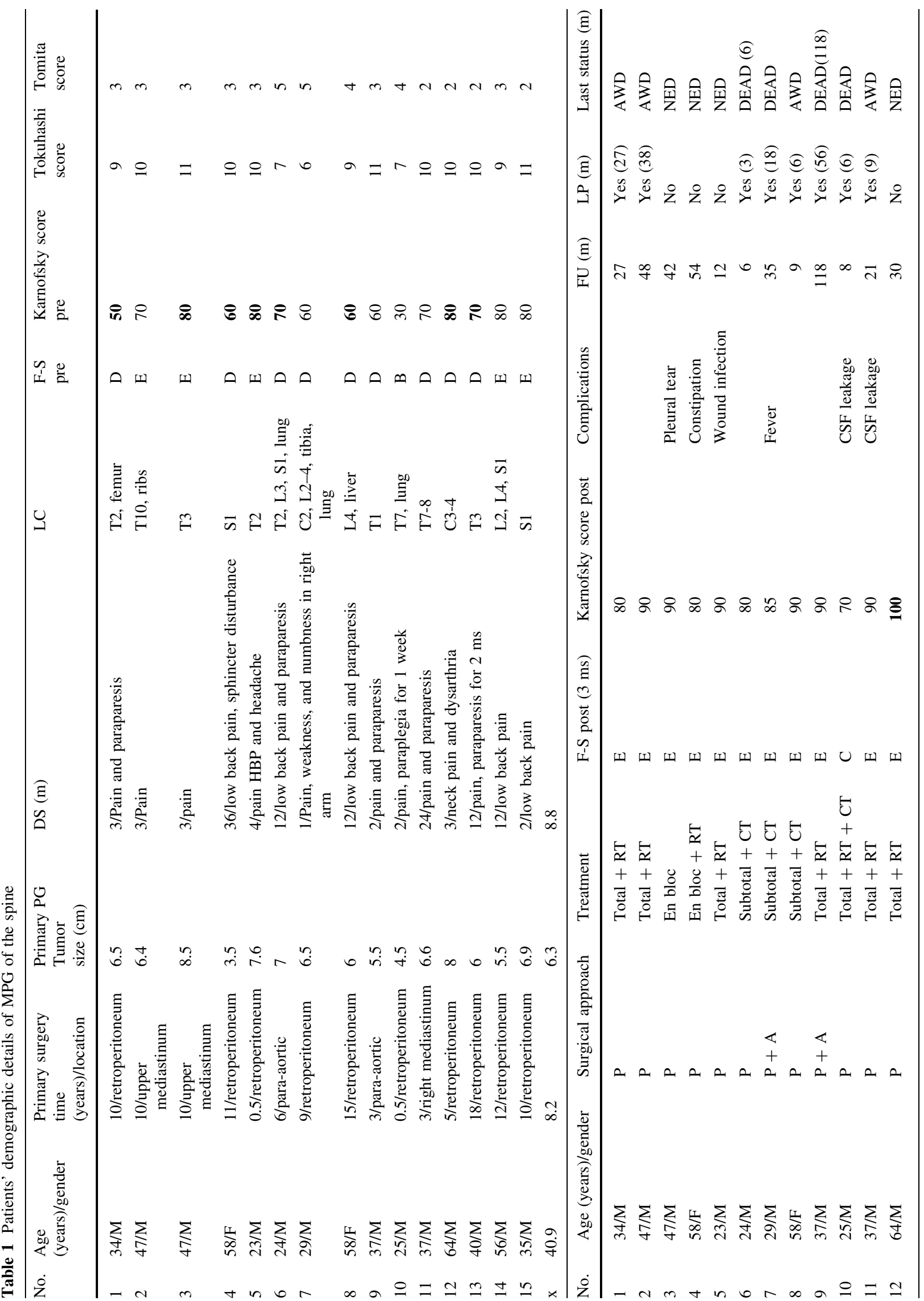




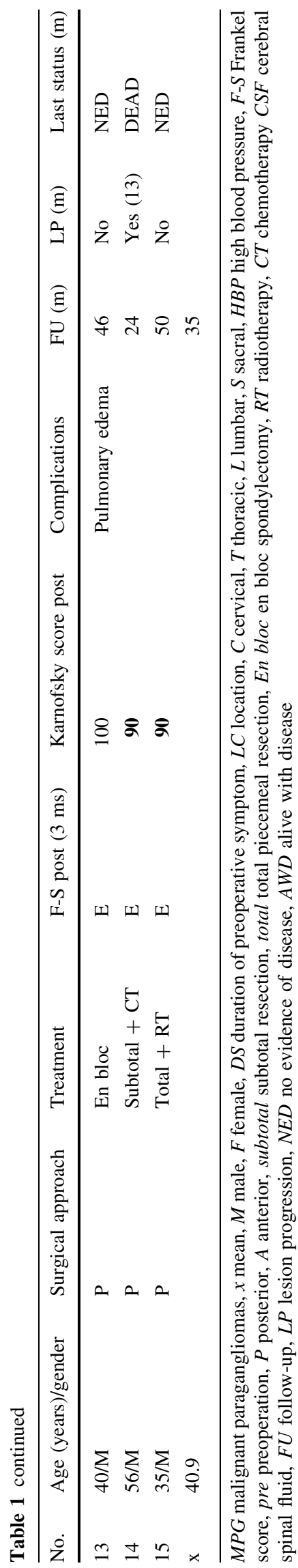

Case 7 and 15), osteosarcoma (Case 6), and neurogenic tumor (Case 10) were misdiagnosed on preoperative MR images. Hypointense or iso-intense signals on $\mathrm{T} 1$ and hyperintense signals on $\mathrm{T} 2$ were common findings (Fig. 2). Functional imaging studies such as FDG-PET/CT were performed in 8 patients with multiple lesions, showing an unusual concentration of 18F-FDG in the lesions. 3 patients underwent bone scans, which was also useful for detecting multiple lesions of the bone.

\section{Treatment and outcome}

Preoperative fine-needle biopsy and intraoperative fast pathologic examination were performed in six and nine patients, respectively, but the results were not all consistent with the final diagnosis of MPG. Only eight intraoperative fast pathologic examination was consistent with the diagnosis of MPG, and the results in the other seven patients suggested the diagnosis of malignancy or metastasis. Noncompetitive alpha-blockade antagonists were given 2 weeks before surgery in patient (Case 5) who showed catecholamine-related symptoms to control hypertensive crisis during surgery. Selective arterial embolization was used through digital subtraction arteriography (DSA) 24-48 $\mathrm{h}$ before surgery in eight patients whose lesions occurred below $\mathrm{T} 4$ in our center.

For solitary metastatic lesion, we preferred total resection including piecemeal total resection and total en bloc spondylectomy (TES). TES was performed in three patients in our series including one patient with contaminated margins that supplemented with posterior adjuvant radiotherapy. Eight patients received piecemeal total resection to remove the bone lesions to the maximum extent assisted with local radiotherapy and for the lung metastasis (Case 10) that could not be surgically removed, chemotherapy was added. Decompression and reconstruction surgery was applied to four patients with multiple metastasis combined with cyclophosphamide-dacarbazine based regimens chemotherapy according to the Tokuhashi and Tomita scoring system. The mean PFS time for TES, piecemeal total, and subtotal resection cases was 47.3 (range 42-54), 28.5 (range 6-56) and 10 (range 3-18) months, respectively (Fig. 1b). Intraoperative blood loss ranged from 600 to 3300 (mean 1978) ml. All surgical procedures were performed uneventfully without severe blood pressure fluctuations. The local pain symptom resolved or disappeared in all patients after surgical treatment. The neurological status at 3-month follow-up showed that the Frankel score improved by 1-2 grades. Catecholamine-related symptoms in Case 5 including the unusual hypertension and headache disappeared immediately after operation, and showed no relapse during the follow-up period. 
Fig. 1 Graphs of survival curve showing the progression-free survival in different metastatic classification (a) and resection mode (b)

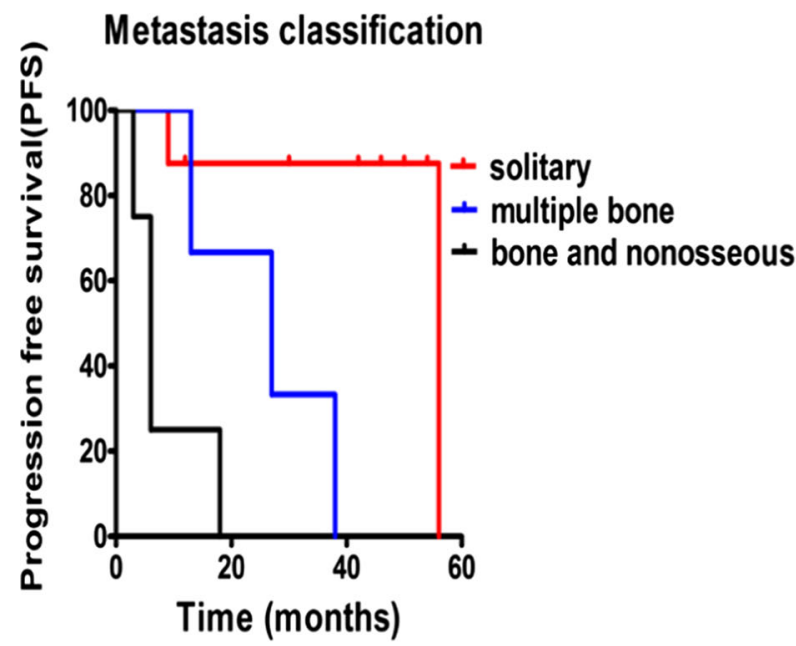

A

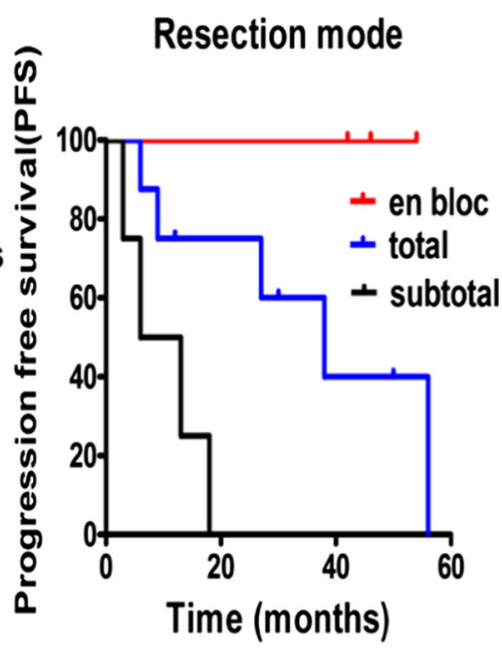

B
The treatment modalities and outcomes for nine lesion progressive patients are listed in Table 2. Two patients selected the revision surgery at least once in our center (Case 9 and 11). Case 2 who had the T3 vertebrae metastasis again accepted the revision surgery combined with the adjuvant radiotherapy. Conservative treatments, including cyclophosphamide-dacarbazine based chemotherapy, local radiotherapy and analgesics (such as Morphine), were applied to the other six patients to alleviate the symptoms and prolong the lives.

\section{Pathology}

Histologic diagnosis was obtained in all our cases. In patients receiving TES, all cutting margins (gross normal surrounding tissue) were submitted for pathologic examination at the same time and 1 of 3 showed contamination margins. Histo-morphologic features of the MPG were similar to the primary PG. Immunohistochemical study showed that the lesions were mostly positive in Vimentin, CD56, chromogranin A, NSE, S-100, synaptophysin and Ki-67 and negative in cytokeratin, epithelial membrane antigen and CK-pan. The value of Ki-67 was commonly positive with a range of $3-30 \%$ and it increased in three patients who had revision surgery for the progressive spinal lesions.

\section{Discussion}

PGs are slow-growing tumors with the risk of malignant transformation [25] which require long-term clinical and radiographic follow-up (MRI and CT scan). Unlike other malignant cancers with lung or liver being the first metastatic organ, the most common site for MPG was skeleton
[10], especially spine, and in our cases nine patients showed the spinal metastasis only (including two multiple metastasis) and six patients presented with multiple metastasis. The reasons that PG cells were inclining to spread to the skeleton were still unclear, but the homing mechanism has been proposed because of strong CXCR4 and CCR9 expression [10, 23].

Although spine is the most frequently metastatic site, due to the low incidence of the primary PGs and only $10-17 \%$ of them evolving to metastasis, reports about MPG involving the spine are quite rare and the epidemiological features, treatment protocols and prognosis are ambiguous. Montserrat Ayala-Ramirez et al. [10] had reported that the median OS was 12 years for patients with only bone metastasis, whereas 5 years for patients with both bone and nonosseous metastases which indicated the better prognosis for bone metastatic patients. Because of the complex anatomical constraints and sometimes lesionrelated spinal cord injured symptoms (paraplegia or paraparesis), the treatment for spinal lesions was tougher than extra-axis bone lesions. In this study, we analyzed the clinical data of 15 cases of spinal MPG and reported our treatment experience which could provide more information about this disease. To the best of our knowledge, this is the largest cohort of patients with spinal MPG so far.

Primary PGs usually arise in the fifth or sixth decade of life [4], but our cohort had a broad age demographic structure with a mean age of 40.9 (range 23-58) years. In our series, $11(73.3 \%)$ of the 15 patients were below 50 years which seems to indicate that spinal MPG is more likely to occur in young patients with PG treatment history. This is an interesting phenomenon worthy of further research. We also found male gender predominance (13:2), which is similar to primary PG [17]. Retroperitoneal PG showed easier later metastasis than other primary PG, 


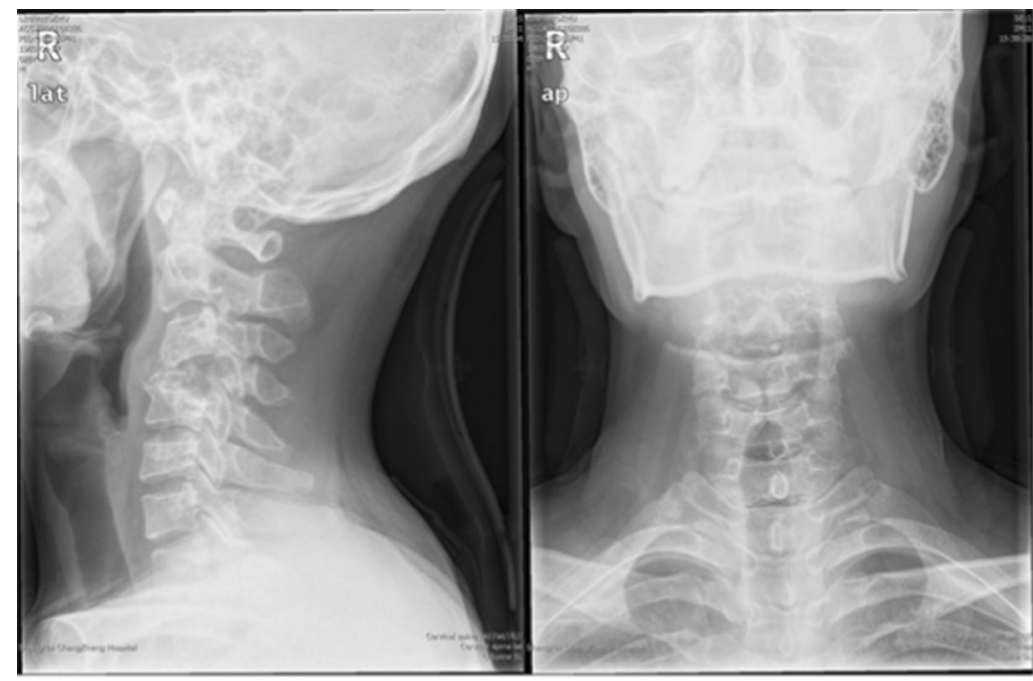

A

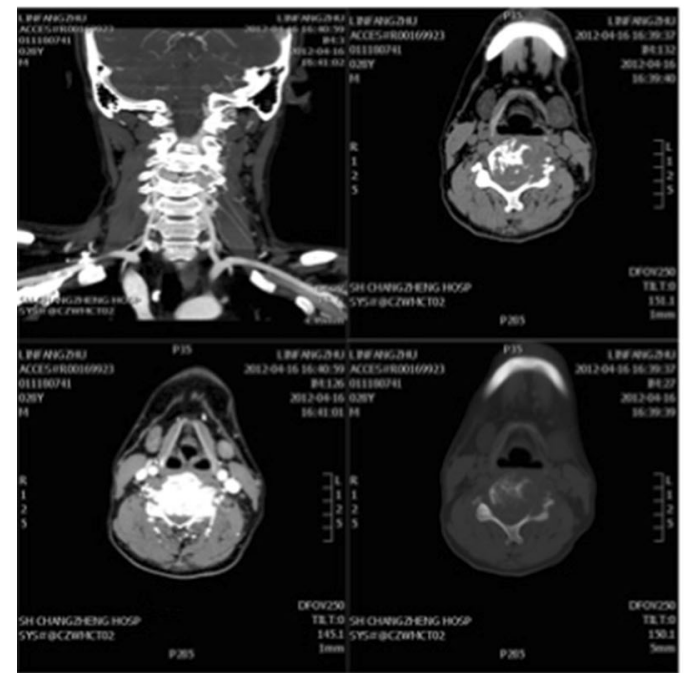

C

Fig. 2 Radiologic images (Case 12). a Preoperative X-ray: an osteolytic lesion in C4; b MRI images: hyperintense in T2 and enhancement after gadolinium injection in C3-4; c CT images: lytic

accounting for $66.7 \%$ of all our cases. Metastasis could be presented at the time of diagnosis of the PG or years later $[7,14]$ and the mean duration from surgical resection of the primary tumor to spinal metastasis was $8.2(0.5-15)$ years. As a result, long-term follow-up was recommended for patients who had the PG treatment history, especially retroperitoneal ones. With respect to location, spinal MPG was most likely to infringe up on the thoracic spine in our series $(53.3 \%)$, which is in line with previous studies in the literature [24].

Spine MPG usually induces intractable local pain and put patients at risk of spinal cord compression [13, 17, 25]. In our cases, local pain is the most common symptom and ten patients $(66.7 \%)$ presented with varying degrees of cord

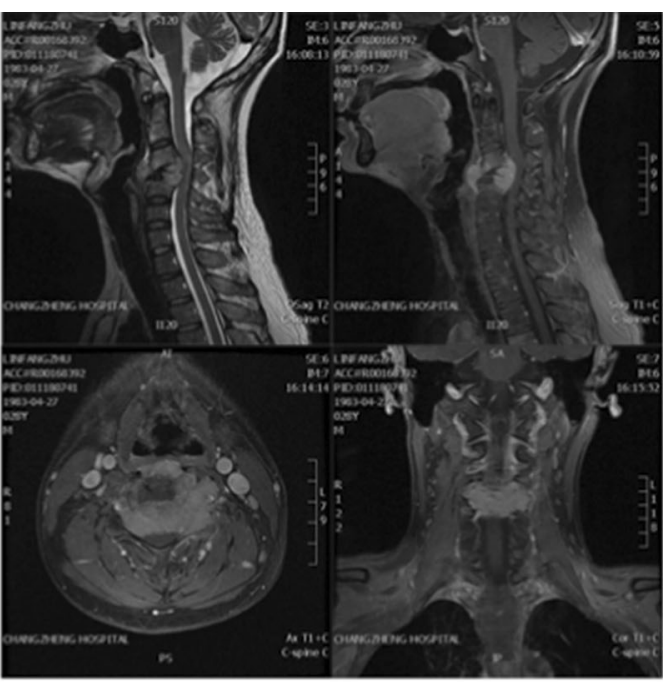

B

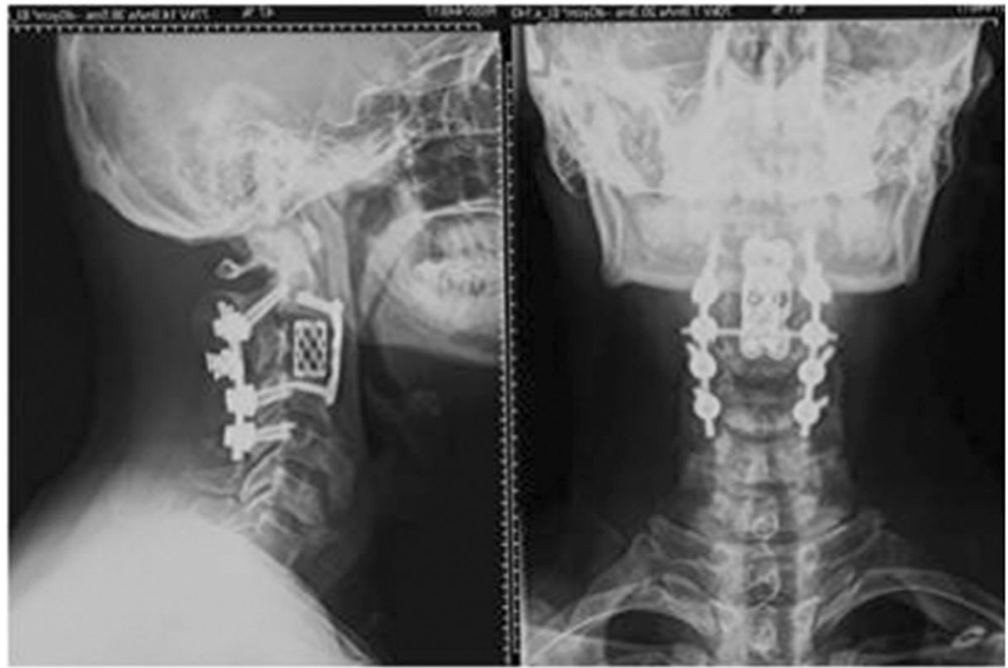

D

changes and compression fracture; d Postoperative X-ray: reconstruction by anterior titanium mesh and plate and posterior screw-rod system from $\mathrm{C} 2$ to $\mathrm{C} 6$

compression at diagnosis. Less frequently, spinal MPG could present with catecholamine-related symptoms, such as elevated blood pressure, diaphoresis, and headache [15] and only one patient (Case 5) showed these symptoms which indicated the low incidence of the catecholamine release for spinal MPG. The radiographic appearance (Xray, CT, MRI) of spinal MPG is nonspecific and somewhat similar to that of other lytic bone lesions; however, certain findings can be integral in establishing the diagnosis in the setting of primary PG surgery history. Compression fracture was common in our cases (66.7\%). 18F-FDG-PET/CT has been used for diagnosis of various neoplasms including MPG [26]; it is also the most effective method to evaluate the metastasis of PGs [10]. 
Table 2 Treatment and outcomes for progressive lesions

\begin{tabular}{|c|c|c|c|c|c|c|c|}
\hline $\begin{array}{l}\text { Case } \\
\text { no. }\end{array}$ & $\begin{array}{l}\text { Recurrent } \\
\text { Location }\end{array}$ & $\begin{array}{l}\text { recurrent } \\
\text { time }(\mathrm{m})\end{array}$ & $\begin{array}{l}\text { Previous } \\
\text { resection mode }\end{array}$ & Treatment strategies & $\begin{array}{l}\mathrm{FU} \\
(\mathrm{m})\end{array}$ & $\begin{array}{l}\text { Last } \\
\text { status (m) }\end{array}$ & Reasons of death \\
\hline 1 & $\mathrm{~T} 2$ & 27 & Total & RT & 27 & AWD & \\
\hline 2 & $\mathrm{~T} 3$ & 38 & Total & Surgery and RT & 48 & AWD & \\
\hline 6 & L3 & 3 & Subtotal & $\mathrm{RT}$ and $\mathrm{CT}$ & 6 & $\begin{array}{l}\text { DEAD } \\
(6)\end{array}$ & Severe pneumonia \\
\hline 7 & $\mathrm{C} 2-3$ & 18 & Subtotal & $\mathrm{RT}, \mathrm{CT}$ and analgesics & 35 & DEAD & Multiple metastasis \\
\hline 8 & $\mathrm{~L} 4$ & 6 & Subtotal & $\mathrm{RT}$ and $\mathrm{CT}$ & 9 & AWD & \\
\hline 9 & $\mathrm{~T} 1$ & 56 & Total & $\begin{array}{l}56 \text { months recurred (T1)-revision } \\
\text { surgery } \\
72 \text { months recurred (T1-2)-revision } \\
\text { surgery and AT } \\
116 \text { months recurred-conservative } \\
\text { treatment }\end{array}$ & 118 & $\begin{array}{r}\text { DEAD } \\
(118)\end{array}$ & $\begin{array}{l}\text { Spinal cord compression } \\
\text { and paralysis }\end{array}$ \\
\hline 10 & $\mathrm{~T} 7$ & 6 & Total & RT & 8 & DEAD & Pulmonary embolization \\
\hline 11 & $\mathrm{~T} 7-8$ & 9 & Total & Revision surgery and RT & 21 & AWD & \\
\hline 14 & L2 & 13 & Subtotal & RT and CT & 24 & DEAD & Multiple metastasis \\
\hline
\end{tabular}

$C$ cervical, $T$ thoracic, $L$ lumbar, $S$ sacral, subtotal subtotal resection, total total piecemeal resection, $R T$ radiotherapy, $C T$ chemotherapy, $F U$ follow-up, $A W D$ alive with disease

There is no doubt that a preoperative biopsy can facilitate surgery protocol formulation, especially for the solitary lesion. Thus, it is regrettable that patients forego it based on fear of possible nerve damage. Surgical treatment is the foundational treatment for spinal MPG. Adequate catecholamine blockage and an excellent anesthesia are necessary to conduct an uneventful surgery, especially for MPG secreting catecholamines [14]. The strategy must be carried on the adequate study of the tumors according to the Tokuhashi and Tomita scoring system. Alexander Richter et al. [6] had reported TES and reconstruction of a solitary spinal MPG in L1 vertebral body with a follow-up time for 10 years which indicated the significance of TES. As a result, with surgical and internal fixation techniques rapidly improving, total resection, even TES when possible, is necessary to treat spinal MPG, especially solitary lesion, to achieve satisfying results. In some cases, an ideal en bloc resection could not be achieved unless the spinal cord and nerve root are killed or multiple metastasis that could not be removed totally, piecemeal total resection or curettage was conducted combined with adjuvant radiotherapy and/or chemotherapy. The mean PFS time for piecemeal total resection combined with radiotherapy was lower than TES and, however, higher than subtotal resection which may indicate that it is an alternative choice when multiple bone metastasis occurred which could not be removed by TES. For bone and nonosseous metastatic patients, although the neurologic status improved and intractable pain resolved after decompression surgery, the prognosis was not satisfied with the mean follow-up time of 18.5 (range 6-35) months. Therefore, although the lesions were metastasis, TES for solitary spinal MPG could result in satisfied prognosis and piecemeal total resection with postoperative radiotherapy can be an acceptable alternative therapy when en bloc resection is not feasible.

Immunohistochemically, there were no reliable markers to predict the malignant potential of PG [12]. Lui et al. [27] found that the Ki-67 index ( $>3 \%)$ was a useful marker for predicting malignancy. All our cases showed the index of Ki-67 higher than 3\%, and as high as $30 \%$. Also, for the progressive spinal MPG, 3 cases receiving revision surgery showed the increase of Ki-67 index which may indicate the positive association between the Ki-67 and the possibility of metastasis. In our cohort, 13 primary PG specimens were bigger than $5 \mathrm{~cm}$ in maximum diameter which accorded with reports that bigger than $5 \mathrm{~cm}$ was more likely to transfer to malignancy and metastasis [7, 10].

Radiotherapy and chemotherapy using as adjuvant treatment for spinal MPG were usually advocated [2, 12, 29, 30]. Radiotherapy with a total of 30-50 Gy had been reported to be helpful for the treatment of spinal MPG [2, 6, 12, 15, 30]. Mertens et al. [28] had reported initial chemotherapy including cyclophosphamide, vincristine and dacarbazine could alleviate symptoms at metastatic sites. Patel et al. [29] proposed the clinically beneficial in MPG patients by cyclophosphamide-dacarbazine based regimens in spite of no obvious benefits in OS. As a result, although litter information could be concluded from our study, for the spinal MPG, especially multiple metastatic ones, radiotherapy and chemotherapy were helpfully supplementary therapy.

The limitation of the present study is its retrospective nature, being performed about ten years. Surgical 
techniques and various kinds of radiotherapy and chemotherapy have been considerably more developed during the follow-up period which may aid in safely accomplishing tumor removal and improve the prognosis. Additionally, the number of our study was too small to conduct the statistic analysis. Although 15 cases appear to be a small number for the study of metastatic lesions, the present cases constitute the first reported clinical series concentrating on surgery-based treatment and prognosis of spinal MPG which may aid the improvement of treatment of this rare disease.

\section{Conclusion}

MPG in the spine is rare, and pain in the involvement site is the most common complaint. The cases presented in the current study highlight the crucial role of surgery. TES for solitary spinal MPG could result in a satisfying prognosis and piecemeal total resection with postoperative radiotherapy should be an alternative therapy when en bloc resection is not feasible. Radiotherapy and chemotherapy were advocated, especially for the multiple metastasis. Long-term follow-up trials are mandatory for patients who have primary PG surgical treatment history especially for the retroperitoneal ones. For spinal MPG patients who present with catecholamine-related symptoms, surgery is also useful to alleviate the symptoms to a great extent.

Acknowledgements This work was supported by the China Scholarship Council (No. 201603170230).

\section{Compliance with ethical standards}

Conflict of interest No conflict of interest exists in the submission of this manuscript for all authors, and manuscript is approved by all authors for publication.

Open Access This article is distributed under the terms of the Creative Commons Attribution 4.0 International License (http://crea tivecommons.org/licenses/by/4.0/), which permits unrestricted use, distribution, and reproduction in any medium, provided you give appropriate credit to the original author(s) and the source, provide a link to the Creative Commons license, and indicate if changes were made.

\section{References}

1. DeLellis RLR, Heitz P, Eng C (2004) Pathology and genetics: tumours of endocrine organs (IARC WHO classification of tumours). IARC Press, Lyon

2. Simpson LN, Hughes BD, Karikari IO et al (2012) Catecholamine-secreting paraganglioma of the thoracic spinal column: report of an unusual case and review of the literature. Neurosurgery 70(4):E1049-E1052 (discussion E52)

3. Lehmen JA, Babbel DM, Mikhitarian K, Choma TJ (2010) Paraganglioma presenting as metastatic lesion in a cervical vertebra: a case report and review of the literature. Spine (Phila Pa 1976) 35(5):E152-E154

4. Lau D, La Marca F, Camelo-Piragua S, Park P (2013) Metastatic paraganglioma of the spine: case report and review of the literature. Clin Neurol Neurosurg 115(9):1571-1574

5. Andersen KF, Altaf R, Krarup-Hansen A et al (2011) Malignant pheochromocytomas and paragangliomas - the importance of a multidisciplinary approach. Cancer Treat Rev 37(2):111-119

6. Richter A, Halm HF, Lerner T, Liljenqvist UR, Quante M (2011) Long-term follow-up after en bloc resection and reconstruction of a solitary paraganglioma metastasis in the first lumbar vertebral body: a case report. J Med Case Rep 5:45

7. Plouin P, Fitzgerald T Rich et al (2012) Metastatic pheochromocytoma and paraganglioma: focus on therapeutics. Horm Metab Res 44:390-399

8. Broggini Thomas, Czabanka Marcus, Piffko Andras et al (2015) ICAM1 depletion reduces spinal metastasis formation in vivo and improves neurological outcome. Eur Spine J 24:2173-2181

9. Zheng H, Li W, Kang Y (2017) Tumor-stroma interactions in bone metastasis: molecular mechanisms and therapeutic implications. Cold Spring Harb Symp Quant Biol. doi:10.1101/sqb. 2016.81.030775

10. Montserrat Ayala-Ramirez J, Palmer Lynn, Hofmann MarieClaude et al (2013) Bone metastases and skeletal-related events in patients with malignant pheochromocytoma and sympathetic paraganglioma. J Clin Endocrinol Metab 98(4):1492-1497

11. Razakaboay M, Maillefert JF, Wendling D et al (1999) Bone metastases from a paraganglioma. A review of five cases. Rev Rhum Engl Ed 66(2):86-91

12. Brodkey JA, Brodkey JS, Watridge CB (1995) Metastatic paraganglioma causing spinal cord compression. Spine (Phila $\mathrm{Pa}$ 1976) 20(3):367-372

13. Houten JK, Babu RP, Miller DC (2002) Thoracic paraganglioma presenting with spinal cord compression and metastases. J Spinal Disord Tech 15(4):319-323

14. Noorda RJ, Wuisman PI, Kummer AJ, Winters HA, Rauwerda JA, Egeler-Peerdeman SM (1996) Nonfunctioning malignant paraganglioma of the posterior mediastinum with spinal cord compression. A case report. Spine (Phila Pa 1976) 21(14):1703-1739

15. Lazaro B, Klemz M, Flores MS, Landeiro JA (2003) Malignant paraganglioma with vertebral metastasis: case report. Arq Neuropsiquiatr 61(2B):463-467

16. Plouin PF, Fitzgerald P, Rich $T$ et al (2012) Metastatic pheochromocytoma and paraganglioma: focus on therapeutics. Hormone and Metabolic Research Hormon und Stoffwechselforschung Hormones et Metabolisme 44(5):390-399

17. Constantini S, Soffer D, Siegel T, Shalit MN (1989) Paraganglioma of the thoracic spinal cord with cerebrospinal fluid metastasis. Spine (Phila Pa 1976) 14(6):643-645

18. Zoccali C, Skoch J, Walter CM et al (2016) The Tokuhashi score: effectiveness and pitfalls. Eur Spine J 25(3):673-678. doi:10. 1007/s00586-015-4339-6

19. Tomita K, Kawahara N, Kobayashi $T$ et al (2001) Surgical strategy for spinal metastases. Spine (Phila $\mathrm{Pa}$ 1976) 26(3):298-306

20. Frankel HL, Hancock DO, Hyslop G et al (1969) The value of postural reduction in the initial management of closed injuries of the spine with paraplegia and tetraplegia: I. Paraplegia 7(3):179-192

21. Karnofsky DA, Burchenal JH (1949) The clinical evaluation of chemotherapeutic agents in cancer. In: MacLeod CM (ed) Evaluation of chemotherapeutic agents. Columbia University Press, New York, p 196

22. Bernard F, Lemée JM, Lucas O, Menei P (2017) Postoperative quality-of-life assessment in patients with spine metastases 
treated with long-segment pedicle-screw fixation. J Neurosurg Spine 26(6):725-735. doi:10.3171/2016.9.SPINE16597

23. DahiaPL RossKN, Wright ME et al (2005) A HIF1 regulatory loop links hypoxia and mitochondrial signals in pheochromocytomas. PLoS Genet 1:72-80

24. Zileli M, Kalayci M, Basdemir G (2008) Paraganglioma of the thoracic spine. J Clin Neurosci 15(7):823-827

25. Sato N, Imai T, Aikawa H et al (2001) Recurrence and pulmonary metastasis of extradural paraganglioma in thoracic vertebral canal: report of a case. Kyobu Geka 54(7):610-613

26. Timmers HJ, Kozupa A, Chen CC et al (2007) Superiority of fluorodeoxyglucose positron emission tomography to other functional imaging techniques in the evaluation of metastatic SDHB-associated pheochromocytoma and paraganglioma. J Clin Oncol 25(16):2262-2269
27. Liu TH, Chen YJ, Wu SF et al (2004) Distinction between benign and malignant pheochromocytomas. Zhonghua bing li xue za zhi Chin J Pathol 33(3):198-202

28. Mertens WC, Grignon DJ, Romano W (1993) Malignant paraganglioma with skeleton metastases and spinal cord compression: response and palliation with chemotherapy. Clin Oncol 5:126-128

29. Patel SR, Winchester DJ, Benjamin RS (1995) A 15-year experience with chemotherapy of patients with paraganglioma. Cancer 76:1476-1480

30. Quraishi NA, Ramoutar D, Sureshkumar D et al (2014) Metastatic spinal cord compression as a result of the unknown primary tumor. Eur Spine J 23:1502-1507 